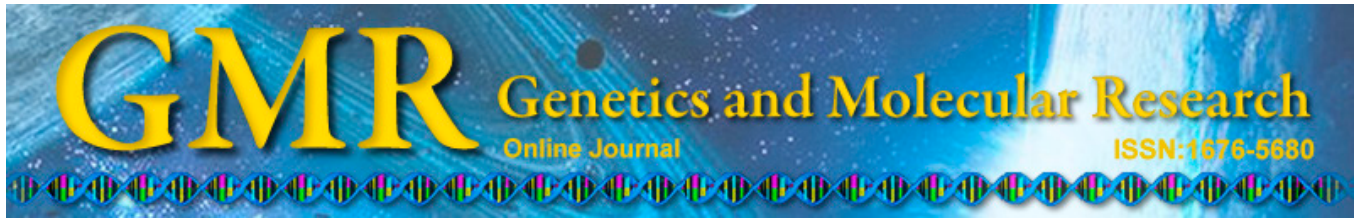

\title{
Mechanism of rat osteosarcoma cell apoptosis induced by a combination of low-intensity ultrasound and 5-aminolevulinic acid in vitro
}

\author{
Y.N. Li ${ }^{1}$, Q. Zhou ${ }^{2}$, B. Yang ${ }^{2}$, Z. Hu ${ }^{2}$, J.H. Wang ${ }^{2}$, Q.S. Li ${ }^{3 *}$ and W.W. Cao ${ }^{1,4 *}$ \\ ${ }^{1}$ School of Life Science and Technology, Harbin Institute of Technology, \\ Harbin, China \\ ${ }^{2}$ Laboratory of Photo-and Sono-Theranostic Technologies and Condensed \\ Matter Science and Technology Institute, Harbin Institute of Technology, \\ Harbin, China \\ ${ }^{3}$ Cardiovascular Institute, \\ The First Affiliated Hospital of Dalian Medical University, Dalian, China \\ ${ }^{4}$ Materials Research Institute, The Pennsylvania State University, \\ State College, PA, USA \\ *These authors contributed equally to this study. \\ Corresponding author: W.W. Cao \\ E-mail: lyn1009@126.com / gaowenwu_gww@yeah.net
}

Genet. Mol. Res. 14 (3): 9604-9613 (2015)

Received December 18, 2014

Accepted June 8, 2015

Published August 14, 2015

DOI http://dx.doi.org/10.4238/2015.August.14.23

\begin{abstract}
We investigated the killing effect of low-intensity ultrasound combined with 5-aminolevulinic acid (5-ALA) on the rat osteosarcoma cell line UMR-106. Logarithmic-phase UMR-106 cells were divided into a control group, ultrasound group and 5-ALA group. The cell apoptotic rate, production of reactive oxygen species, and the change in mitochondrial membrane potential were analyzed by flow cytometry; ultrastructural changes were observed by transmission electron microscopy. Using low-intensity ultrasound at $1.0 \mathrm{MHz}$ and $2.0 \mathrm{~W} / \mathrm{cm}^{2}$ plus 5 -ALA at a concentration of $2 \mathrm{mM}$, the apoptotic rate of the sonodynamic therapy group was $27.2 \pm 3.4 \%$ which was significantly higher than that of the control group, ultrasound group, and 5-ALA group $(\mathrm{P}<0.05)$. The
\end{abstract}


production of reactive oxygen species was $32.6 \pm 2.2 \%$ and the decrease in mitochondrial membrane potential was $39.5 \pm 2.5 \%$. The 33342 staining showed nuclear condensation and fragmentation in the ultrasound group and 5-ALA group. Structural changes in the cell membrane, mitochondria, Golgi apparatus, and other organelles observed by transmission electron microscopy included formation of apoptotic bodies. The killing effect of low-intensity ultrasound combined with 5-ALA on UMR-106 cells was significant. Cell apoptosis played a vital role in the killing effect, and the mitochondria pathway contributed to the apoptosis of UMR-106 cells.

Key words: 5-Aminolevulinic acid; Apoptosis; Sonodynamic therapy; UMR-106 cells

\section{INTRODUCTION}

Sonodynamic therapy (SDT) is a new tumor treatment method based on photodynamic therapy (Agostinis et al., 2011). Recently, following the development of ultrasonic medicine, studies on the biological effects have shown breakthrough progress, including combined treatment with low-intensity ultrasound and a sonosensitizer at the tissue, cell, and molecular levels. Previous studies showed that using SDT as a tumor treatment induced irreversible injury of tumor cells using ultrasound and ultrasonic medicine (Tang et al., 2010; Li et al., 2012). Ultrasonic therapy kills tumor cells, an effect that is specific to the tumor location and is retained for a long period, causing little damage to the adjacent normal tissues. Thus, this therapy has a wide range of potential applications.

5-Aminolevulinic acid (5-ALA) is a common sonosensitizer used in SDT. 5-ALA itself does not show sound sensitivity, but it can be converted to protoporphyrin IX (PpIX). PpIX is a strong endogenous sonosensitizer and exhibits advantages such as a shorter period of skin photosensitivity, low phototoxicity to normal tissues, and rapid elimination from tissues. A study revealed higher levels of porphyrins in tumor tissues than in normal tissues, indicating that specific selection treatment could be used (Tang et al., 2010). Another study revealed that the tumor cytotoxicity induced by ultrasound combining with the ultrasonic medicine was higher than that using ultrasound alone (Peng et al., 1997).

Osteogenic sarcoma is the most common type of primary malignancy. Therapeutic treatments include surgical operation, radiotherapy, chemotherapy, and comprehensive treatments. This tumor shows a high degree of malignancy and poor prognosis, particularly in adolescents (Liu et al., 2008). Thus, it is important to develop new treatments for osteogenic sarcoma in order to improve its cure rate. In this study, we investigated the killing effect and mechanism of low-intensity ultrasound and 5-ALA combination treatment on the rat osteosarcoma cell line UMR-106.

\section{MATERIAL AND METHODS}

\section{Materials}

\section{Cells}

Rat osteosarcoma UMR-106 cells were purchased from Cell Bank, Shanghai Institutes for Biological Science of Chinese Academy of Sciences (Shanghai, China). 


\section{Reagents and instruments}

5-ALA (Sigma, St. Louis, MO, USA) was prepared in phosphate-buffered saline and filtered to remove bacteria, then preserved at $4^{\circ} \mathrm{C}$ in the dark. Dimethyl sulfoxide (Sigma), rhodamine (Sigma), and an annexin-V-fluorescein isothiocyanate/propidium iodide apoptosis kit (KeyGEN BioTECH, Nanjing, China) were used. An automated immunoanalyzer (BioRad, Hercules, CA, USA), fluorescence spectrophotometer (Bio-Rad), and flow cytometer (BD Biosciences, Franklin Lakes, NJ, USA) were also used.

\section{Ultrasonic therapeutic apparatus}

The ultrasound generator and power amplifier were independently developed by Harbin Institute of Technology University (Heilongjiang, China). The ultrasonic transducer (diameter: $3.5 \mathrm{~cm}$; resonance frequency: $1.0 \mathrm{MHz}$; duty cycle: $10 \%$; repetition rate: $100 \mathrm{~Hz}$ ) was built in our laboratory; the ultrasound radiation frequency was $1 \mathrm{MHz}$ and the power was 2 $\mathrm{W} / \mathrm{cm}^{2}$ (Figure 1).

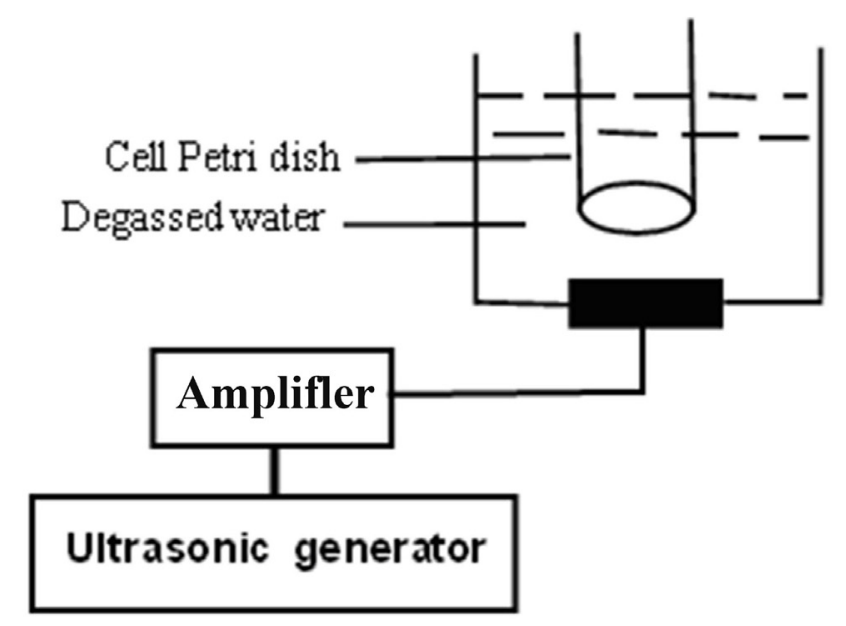

Figure 1. Ultrasound device used for cell treatment.

\section{Methods}

\section{Cell culture, groups, and 5-ALA metabolism}

Rat osteosarcoma UMR-106 cells were cultured in fetal bovine serum at $37^{\circ} \mathrm{C}$ in a humidified atmosphere of 5\% $\mathrm{CO}_{2}$ and passaged once every 2-3 days. Cells were subcultured when cells were in the logarithmic growth phase. Next, cells were seeded on 6-well plates at a density of $1 \times 10^{5}-0.5 \times 10^{5}$ cells/well. They were randomly divided into 4 groups: control group, 5-ALA group, ultrasound group, and ultrasound+5-ALA (SDT) group. Each group included 8 plates. 
Selection of optimal concentration and incubation time of 5-ALA on UMR-106 using MTT method and fluorescence spectrophotometry

The 5-ALA concentration was selected after testing $0.5,1.0,1.5,2.0,3.0$, and $4.0 \mathrm{mM}$ 5-ALA. Incubation times of 2, 4, 6, 8, 10, 12, 16, 20, and $24 \mathrm{~h}$ were tested. Combinations of concentration and time were examined. Each group was tested 6 times, and the optimal concentration and incubation time were selected.

Detection of apoptosis using double labeling with annexin-V-fluorescein isothiocyanate/propidium iodide and flow cytometry

Cells in the logarithmic growth phase were added to 6-well plates, with 8 plates in each group. Next, 5-ALA was added to the wells and incubated for the optimal incubation time. Cells were treated with a fixed ultrasonic frequency of $1.0 \mathrm{MHz}$ and sound intensity of $2.0 \mathrm{~W} / \mathrm{cm}^{2}$. Cells were then cultured at $37^{\circ} \mathrm{C}$ in a humidified atmosphere of $5 \% \mathrm{CO}_{2}$ after ultrasonic irradiation for $12 \mathrm{~h}$. Next, $5 \mu \mathrm{L}$ annexin-V-fluorescein isothiocyanate was added to the wells and the cells were then incubated for $15 \mathrm{~min}$ in the dark; this was followed by addition of $10 \mu \mathrm{L}$ propidium iodide and incubation for $5 \mathrm{~min}$ in the dark. Apoptosis was detected by flow cytometry.

Detection of changes in mitochondrial membrane potential (MMP) using rhodamine123

After ultrasonic irradiation for $12 \mathrm{~h}, 200 \mathrm{nM}$ rhodamine 123 was added to the 4 cell groups, which were then cultured in an incubator for $30 \mathrm{~min}$ in the dark. Changes were detected by flow cytometry.

\section{Detection of reactive oxygen species (ROS) using dichloro-dihydro-fluorescein- diacetate}

Dichloro-dihydro-fluorescein-diacetate was added to the 4 cell groups after ultrasonic irradiation for $2 \mathrm{~h}$ at a final concentration of $10 \mathrm{mM}$. ROS were detected using flow cytometry after culture in an incubator for $30 \mathrm{~min}$ in the dark.

\section{Observation of nuclear using Hoechst33342 fluorescence staining assay}

The 4 groups of cells were washed with phosphate-buffered saline 2 times after ultrasonic irradiation for $24 \mathrm{~h}$, and then fixed in $4 \%$ formaldehyde-phosphate buffer for $15 \mathrm{~min}$ at $4^{\circ} \mathrm{C}$. Cells were stained with $100 \mu \mathrm{L}$ Hoechst 33342 for $10 \mathrm{~min}$ at room temperature. The cells were observed and photographed under a microscope.

\section{Observation of cell ultrastructure by transmission electron microscopy}

The 4 group cells were collected by centrifugation and washed with $0.1 \mathrm{M}$ phosphatebuffered saline, $\mathrm{pH}$ 7.2-7.4. Cells were embedded in agarose; next, the cells were fixed using 
glutaraldehyde and osmium tetroxide. The cells were dehydrated in a graded alcohol series and embedded in Epon812. Samples were prepared by conventional ultrathin sections, stained with lead citrate, and observed by transmission electron microscopy and photographed.

\section{Statistical analysis}

Statistical analysis was performed using the SPSS version 13.0 software (SPSS, Inc., Chicago, IL, USA). Data are reported as means \pm SD. One-way analysis of variance was used to compare groups. Differences of $\mathrm{P} \leq 0.05$ were considered to be statistically significant.

\section{RESULTS}

\section{Optimal concentration and incubation time of 5-ALA on UMR-106 using MTT method and fluorescence spectrophotometry}

The results showed that the optical density of PpIX transferred in UMR-106 cells increased over time at $2 \mathrm{mM} 5$-ALA, reaching a maximum value at $6 \mathrm{~h}$. Thus, the optimal incubation time of 5-ALA was $6 \mathrm{~h}$ (Figure 2A-D).

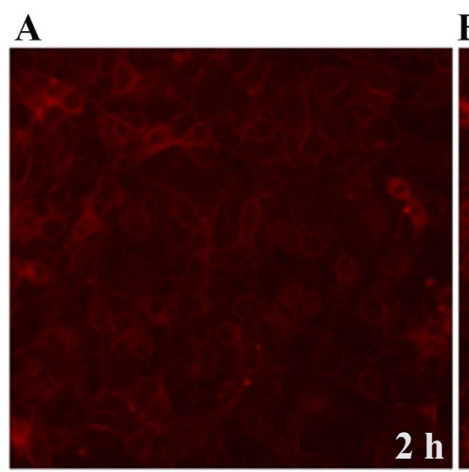

B

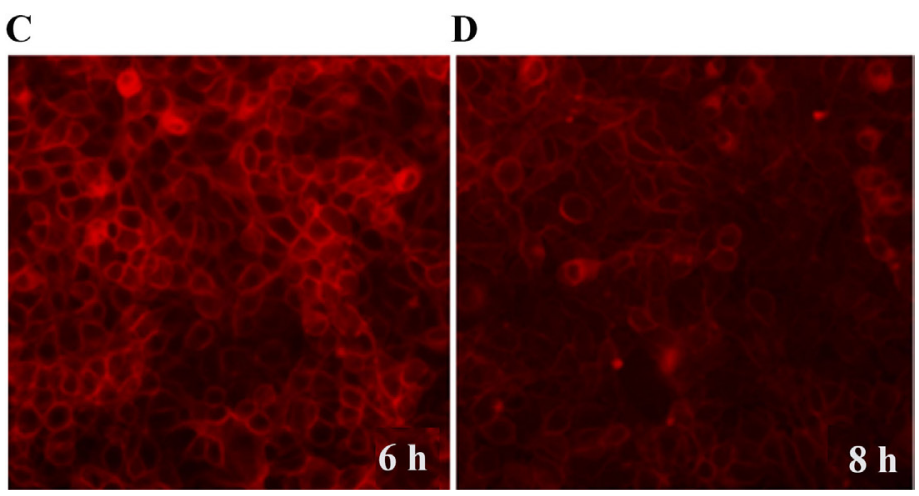

Figure 2. Fluorescence intensity of PpIX in cells at $2 \mathrm{mM} \mathrm{5-ALA} \mathrm{under} \mathrm{a} \mathrm{fluorescence} \mathrm{microscope} \mathrm{(400X);}$ fluorescence intensity was highest at $6 \mathrm{~h}$, and experiments were repeated 6 times. ALA $=5$-aminolevulinic acid; PpIX = protoporphyrin IX. 


\section{Detection of apoptosis using flow cytometry}

The results indicated that some UMR-106 cells had undergone apoptosis and necrosis following single ultrasonic irradiation (frequency: $1 \mathrm{MHz}$; power: $2 \mathrm{~W} / \mathrm{cm}^{2}$ ). The apoptosis rate in the SDT group reached $27.2 \pm 3.4 \%$, which was higher than that in the ultrasound, 5-ALA, and control groups (Figure 3A-B).
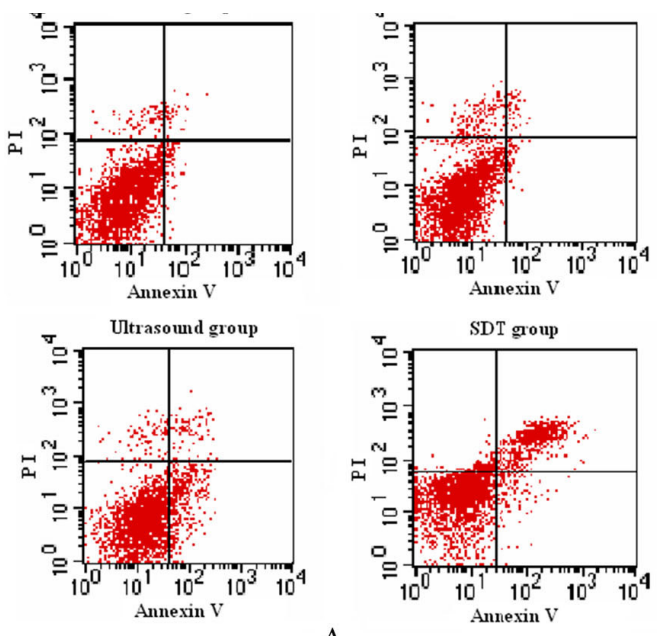

A

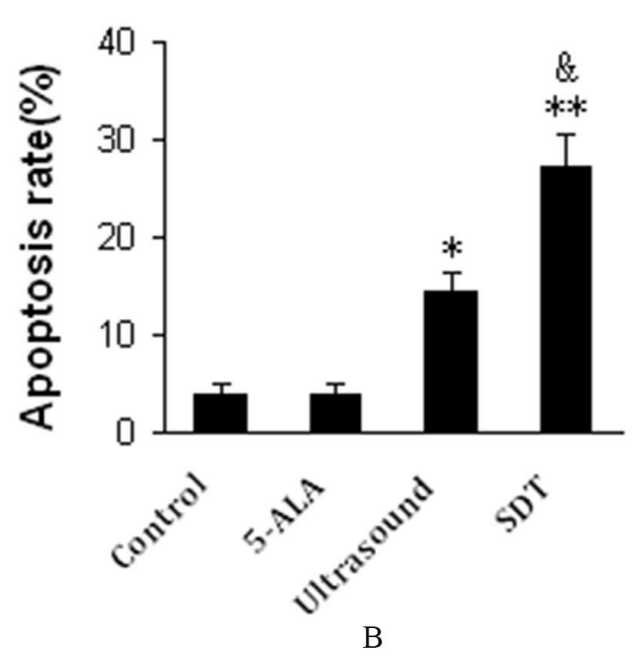

Figure 3. A. Apoptosis and necrosis rates of UMR-106 cells for the control cells and cells treated with $2 \mathrm{mM}$ ALA alone, ultrasound alone ( $7 \mathrm{~min}$ ), or SDT ( $2 \mathrm{mM}$ ALA plus $5 \mathrm{~min}$ ultrasound exposure), measured using flow cytometry with double staining of annexin V and PI. *P $<0.05 ; * * \mathrm{P}<0.01$ compared with the control group; \&P $<0.05$ compared with the ultrasound alone group. ALA $=5$-aminolevulinic acid; PI, propidium iodide; SDT $=$ sonodynamic therapy. B. Detection of apoptosis rate of UMR-106 cells using flow cytometry.

\section{Changes of MMP}

MMP in the ultrasound group and SDT group decreased, but was more significant in the SDT group. The percentage decrease in the SDT group was $39.5+2.5 \%$. There was no significant decrease in the control or 5-ALA group (Figure 4).

\section{ROS production}

The percentages of ROS production in the SDT, control, 5-ALA, and ultrasound groups were $32.6 \pm 2.2,10.4 \pm 1.7,11.8 \pm 1.6$, and $14.3 \pm 1.5 \%$, respectively (Figure 5A-E). There was a significant difference between the SDT group and the other groups $(\mathrm{P}<0.05)$.

\section{Nucleus observation using Hoechst33342 fluorescent staining assay}

The cell morphology was clear, with consistent staining and clear contour boundaries in the control group and 5-ALA group. Cell damage was observed in the ultrasound and SDT groups; the damage extent and degree in the SDT group was significantly higher in these 
groups than in the ultrasound group. Cells in the SDT group showed nuclear chromatin condensation, increased density, and dark staining. The volume of the nucleus was reduced. Some nuclear chromatin was fragmented and was light blue (Figure 6A-D).

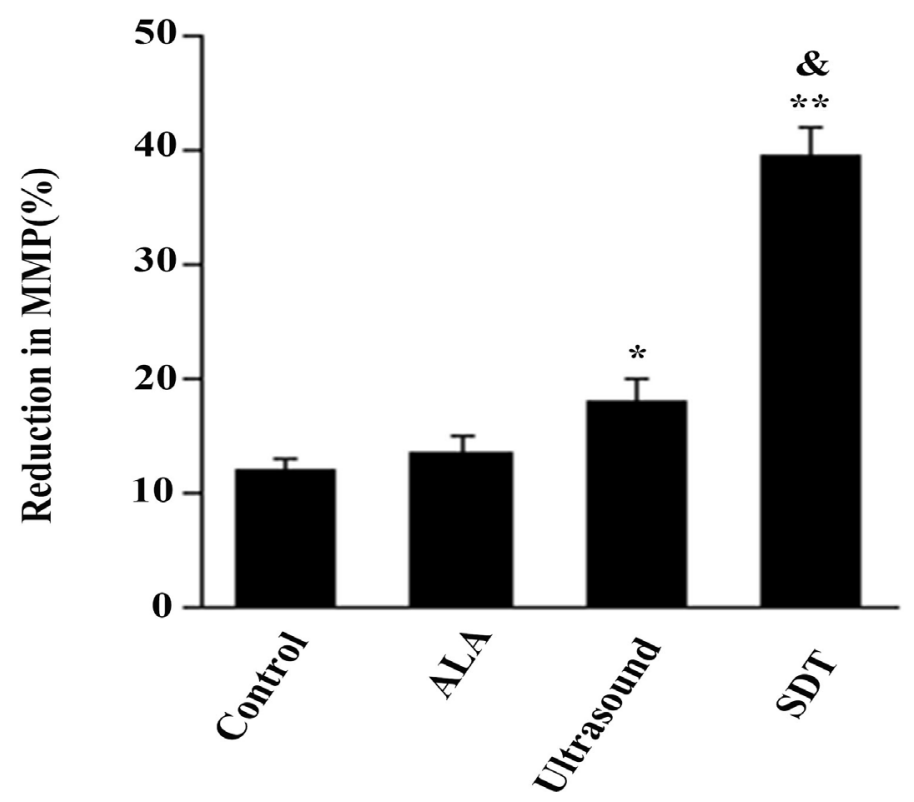

Figure 4. Detection of changes in MMP due to rhodamine123 (SDT group showed a significant difference compared with the other 3 groups, $\mathrm{P}<0.05)$. ${ }^{*} \mathrm{P}<0.05 ; * * \mathrm{P}<0.01$ compared with the control group; ${ }^{\mathrm{P}}<0.05$ compared with the ultrasound alone group.

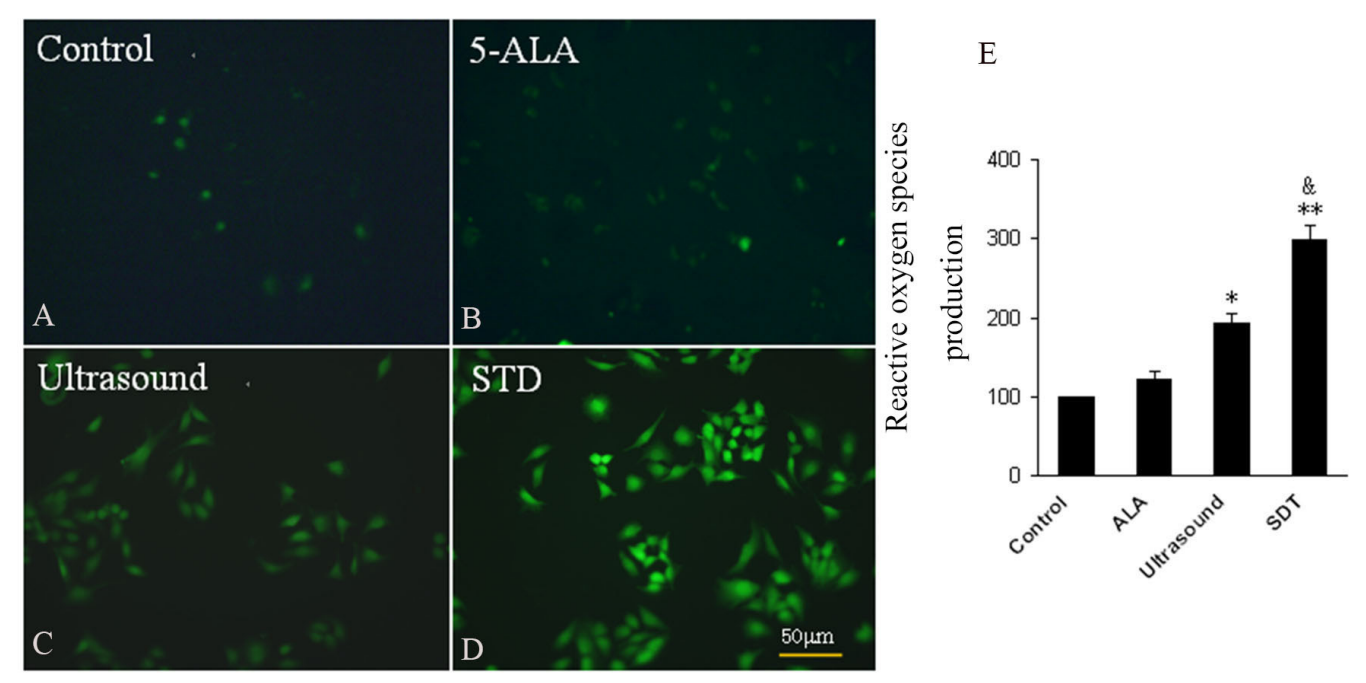

Figure 5. Detection of ROS production by DCFH-DA (SDT group showed a significant difference compared with the other 3 groups, $\mathrm{P}<0.05)$. ${ }^{*} \mathrm{P}<0.05 ; * * \mathrm{P}<0.01$ compared with the control group; ${ }^{\&} \mathrm{P}<0.05$ compared with the ultrasound alone group. 


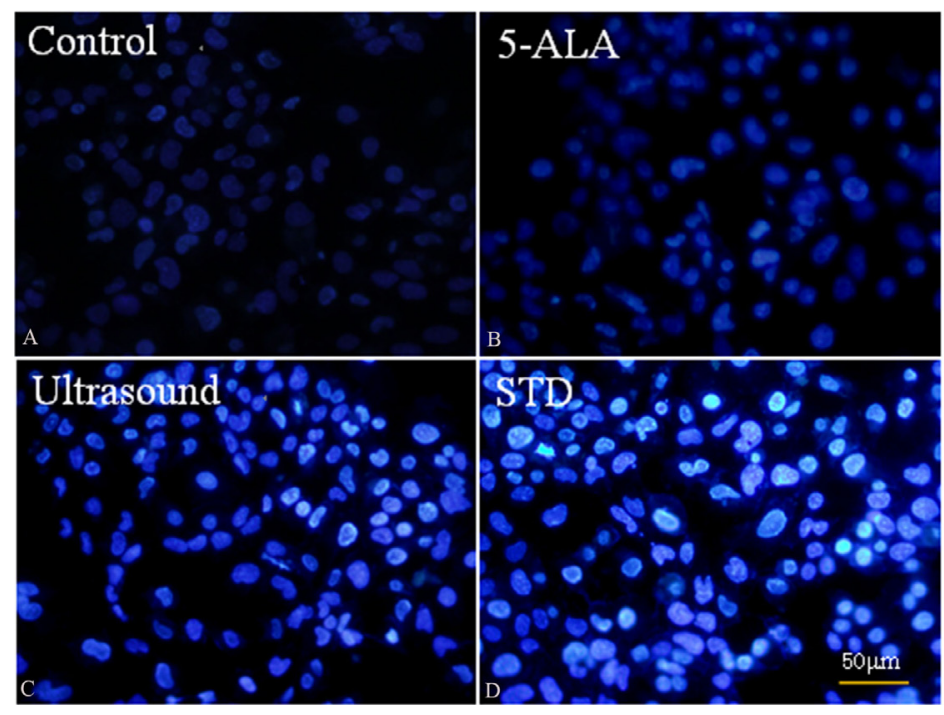

Figure 6. Detection of apoptosis by Hoechst 33342 fluorescent staining assay. Scale bar: $50 \mu \mathrm{m}$. ALA = 5-aminolevulinic acid; PpIX = protoporphyrin IX.

\section{Observation of cell ultrastructure by transmission electron microscopy}

The degree of damage to the structure of UMR-106 cells and apoptotic bodies in the SDT group were significantly higher than in the other groups. In the SDT group, cells varied in size, had irregular shapes, and showed deformities of the membrane, and the microvilli on the cell surface had decreased or disappeared; vacuoles of varying size in the cytoplasm increased and the mitochondria and endoplasmic reticulum were swollen and more significant. Mitochondrion cristae were broken and decreased; the cellular membrane, nuclear membrane, and mitochondria were partly dissolved into pieces. Cell organs in the cytoplasm had begun to decay. The nuclear space increased and the nucleus was fragmented and dissolved; apoptotic bodies were formed (Figure 7A-D).

\section{DISCUSSION}

In this study, we investigated the killing effect and mechanism of SDT mediated by 5-ALA against osteosarcoma UMR-106 cells. Sonosensitizer and ultrasound parameters were important factors for SDT. Compared with other sonosensitizers, 5-ALA has advantages such as low phototoxicity and requirement for a shorter dark period. 5-ALA itself was not an exogenous sonosensitizer, but it was a precursor of the heme, formed in a reaction catalyzed by ALA dehydratase and transformed into PpIX using ultrasound. Normally, ALA synthesis was strictly controlled by negative feedback mechanisms in the body, so there was no excessive accumulation of ALA. In the presence of large amounts of exogenous ALA, the mechanism was interrupted and ALA can be absorbed by tumor cells with vigorous metabolism, in which ALA was transformed into PpIX and accumulated in cells, which was activated by ultrasound to kill tumor cells (Longhi et al., 2006).

At the selected incubation time of 5-ALA, the fluorescence intensity of PpIX at dif- 

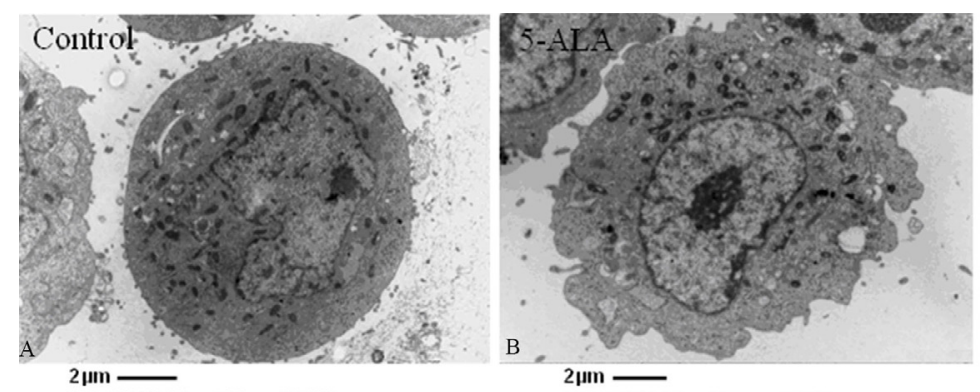
$\mathrm{HV}=80 \mathrm{KV}$ Direct Mag : 12,000X

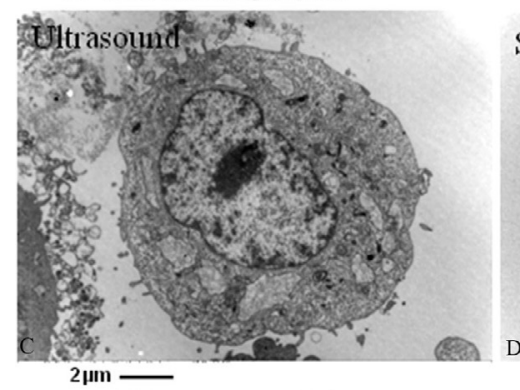

$\mathrm{HV}=80 \mathrm{KV}$ Direct Maa : 12,000x

$\mathrm{HV}=\mathbf{8 0 K V}$ Direct Mag : 12,000x

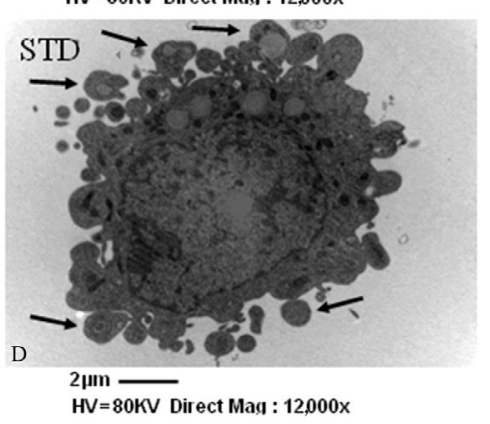

Figure 7. Nuclear fragmentation, dissolution, and apoptotic bodies were observed by TEM (apoptotic bodies indicated by an arrow, 12,000X).

ferent times was observed by fluorescence spectrophotometry (excitation wavelength of 405 $\mathrm{nm})$. The results showed that PpIX produced in UMR-106 cells treated with 5-ALA increased over time until $6 \mathrm{~h}$, and the amount did not increase after $6 \mathrm{~h}$. This indicated that PpIX reached a maximum value at $6 \mathrm{~h}$; under the same conditions, PpIX increased with increase in 5-ALA concentrations, but saturation was observed at $2 \mathrm{mM} 5$-ALA.

In this study, UMR-106 cells were induced to undergo apoptosis using a combination of low-intensity ultrasound and 5-ALA. The results revealed that SDT was effective for inducing apoptosis and significantly better than single ultrasound.

In the apoptosis mechanism induced by SDT, singlet oxygen was produced after the sonosensitizer activated by ultrasound, which had extreme oxidative effects and can destroy the membrane to increase permeability; the mitochondria swelled, the endoplasmic reticulum was broken down, and lysosomal enzyme was released. In this manner, the enzyme activity and nucleic acid structure were affected, contributing to tumor cell death (Mates, 2000; Kinoshita and Hynynen, 2006; Wang et al., 2008, 2010; Li et al., 2012). Additionally, high levels of oxygen free radicals were produced because of ultrasonic cavitation kill tumor cells. We found that the apoptosis of UMR-106 cells was accompanied by the production of ROS, which is produced during the respiration process and is in dynamic equilibrium under normal conditions. The balance between the production and elimination of ROS was disrupted when the cells were induced by physicochemical and biological factors, resulting in high ROS production and apoptosis. ROS increased greatly in the SDT group, suggesting that PpIX in tumor cells activated by ultrasound led to the prominent production of ROS in UMR-106 cells, after which the cell membrane was broken down and permeability increased. Additionally, the production of ROS 
was accompanied by a decrease in MMP, indicating that the high production of ROS damaged the mitochondrial membrane and contributed to permeability increase, MMP decrease, and induced apoptosis (Yue et al., 2009; Wang et al., 2010; Song et al., 2011; Lv et al., 2012).

In conclusion, we observed significant killing effects using a combination of lowintensity ultrasound and 5-ALA on UMR-106 cells; the mechanism underlying this effect was that endogenous apoptosis of cells was induced by breaking down the membrane and mitochondria. Because of this aggregation effect of 5-ALA on tumor cells, tumor cell apoptosis was induced by combination therapy, which included the advantage of low-intensity ultrasound. The killing effect of low-intensity ultrasound combined with 5-ALA on UMR-106 cells was significant. Cell apoptosis played a vital role in the killing effect, and the mitochondrion pathway contributed to the apoptosis of UMR-106 cells.

\section{ACKNOWLEDGMENT}

Research supported by the National Key Technology R\&D Program of China (Grant \#2013BAI03B06).

\section{Conflicts of interest}

The authors declare no conflict of interest.

\section{REFERENCES}

Agostinis P, Berg K, Cengel KA, Foster TH, et al. (2011). Photodynamic therapy of cancer: an update. CA Cancer J. Clin. 61: 250-281.

Kinoshita M and Hynynen K (2006). Mechanism of porphyrin-induced sonodynamic effect: possible role of hyperthermia. Radiat. Res. 165: 299-306.

Li Y, Wang P, Zhao P, Zhu S, et al. (2012). Apoptosis induced by sonodynamic treatment by protoporphyrin IX on MDAMB-231 cells. Ultrasonics 52: 490-496.

Liu Q, Li X, Xiao L, Wang X, et al. (2008). Sonodynamically induced antitumor effect of hematoporphyrin on Hepatoma 22. Ultrason. Sonochem. 15: 943-948.

Longhi A, Errani C, De Paolis M, Mercuri M, et al. (2006). Primary bone osteosarcoma in the pediatric age: state of the art. Cancer Treat. Rev. 32: 423-436.

Lv Y, Fang M, Zheng J, Yang B, et al. (2012). Low-intensity ultrasound combined with 5-aminolevulinic acid administration in the treatment of human tongue squamous carcinoma. Cell Physiol. Biochem. 30: 321-333.

Mates JM (2000). Effects of antioxidant enzymes in the molecular control of reactive oxygen species toxicology. Toxicology 153: 83-104.

Peng Q, Warloe T, Berg K, Moan J, et al. (1997). 5-Aminolevulinic acid-based photodynamic therapy. Clinical research and future challenges. Cancer 79: 2282-2308.

Song W, Cui H, Zhang R, Zheng J, et al. (2011). Apoptosis of SAS cells induced by sonodynamic therapy using 5-aminolevulinic acid sonosensitizer. Anticancer Res. 31: 39-45.

Tang W, Liu Q, Zhang J, Cao B, et al. (2010). In vitro activation of mitochondria-caspase signaling pathway in sonodynamic therapy-induced apoptosis in sarcoma 180 cells. Ultrasonics 50: 567-576.

Wang XB, Liu QH, Wang P, Tang W, et al. (2008). Study of cell killing effect on S180 by ultrasound activating protoporphyrin IX. Ultrasonics 48: 135-140.

Wang XB, Wang P, Tong WY and Liu Q (2010). Comparison of pharmacokinetics, intracellular localization and sonodynamic efficacy of endogenous and exogenous protoporphyrin IX in sarcoma 180 cells. Ultrasonics 50: 803810.

Yue XL, Lehri S, Li P, Barbier-Chassefière V, et al. (2009). Insights on a new path of pre-mitochondrial apoptosis regulation by a glycosaminoglycan mimetic. Cell Death Differ. 16: 770-781. 